\title{
An MD-FE coupling simulation method applied to fracture of viscoelastic-viscoplastic glassy polymers
}

\author{
Wuyang Zhao*, Sebastian Pfaller ${ }^{\dagger}$ \\ Institute of Applied Mechanics (LTM) \\ Friedrich-Alexander-Universität Erlangen-Nürnberg \\ Egerlandstrasse 5, 91058 Erlangen, Germany \\ * e-mail: wuyang.zhao@fau.de \\ $\dagger$ e-mail: sebastian.pfaller@fau.de
}

\begin{abstract}
Fracture of materials is a multiscale process and requires appropriate simulation techniques. In order to obtain fine scale insights into the processes near the crack tip, an atomistic point of view in this region is suitable, but needs to be embedded into macroscopic loading conditions of the surrounding material. In this regard, particle-continuum coupling methods are appropriate for simulations of fracture, which treat the vicinity of the crack at particle resolution while describing the material far away by continuum mechanics.

Although there have been various multiscale simulation methods designed to study the fracture of crystalline materials or elastic polymers, multiscale methods for simulations of viscoelastic or viscoplastic polymers are still rare due to the complex mechanical behavior and the material's amorphous structure. The Capriccio method [1] was proposed for multiscale simulations of glassy polymers within the elastic regime, which couples a particle domain resolved by Molecular Dynamics (MD) simulations with stochastic boundary conditions and a continuum domain solved by the Finite-Element (FE) method. This method has been extended to inelasticity [4] by employing a viscoelastic-viscoplastic constitutive model [3] informed by MD simulations [2] in the continuum domain.

In this contribution, we enhance the inelastic Capriccio method [4] to simulations of fracture of glassy polymers. We consider a polystyrene sample with a pre-crack consisting of a cubic MD domain embedded in an FE domain. The sample is loaded in Mode-I and its mechanical behavior is studied.
\end{abstract}

\section{REFERENCES}

[1] S. Pfaller, M. Rahimi, G. Possart, P. Steinmann, F. Müller-Plathe. and M.C. Böhm. An Arlequin-based method to couple molecular dynamics and finite element simulations of amorphous polymers and nanocomposites. Comput. Methods. Appl. Mech. Eng. (2013) 260: $109-129$.

[2] M. Ries, G. Possart, P. Steinmann, S. Pfaller. Extensive CGMD simulations of atactic PS providing pseudo experimental data to calibrate nonlinear inelastic continuum mechanical constitutive laws. Polymers. (2019) 11:1824.

[3] W. Zhao, M. Ries, P. Steinmann and S. Pfaller. A viscoelastic-viscoplastic constitutive model for glassy polymers informed by molecular dynamics simulations. Int. J. Solids. Struct. (2021): In press.

[4] W. Zhao, P. Steinmann and S. Pfaller. A particle-continuum coupling method for multiscale simulations of viscoelastic-viscoplastic amorphous glassy polymers. Submitted. 